\title{
Feasibility of Time-Frequency Urban Area Analysis on TerraSAR-X Fully Polarimetric Dataset
}

\author{
Salman Khan and Raffaella Guida \\ Dept. of Electronics Engineering \\ Surrey Space Center, University of Surrey \\ Guildford, U.K. \\ Email: \{s.s.khan,r.guida\}@surrey.ac.uk
}

\begin{abstract}
The feasibility of azimuthal Time-Frequency (TF) analysis for urban area identification using TerraSAR-X (TX) fully polarimetric data has been investigated for the first time. Space-borne sensors, unlike airborne sensors, are characterized by a very small azimuth antenna aperture, which limits the SubAperture (SA) decomposition in the azimuth direction. Due to this limitation, the usefulness of SA decomposition for space-borne sensors, has still not been explored. Pixel stationarity, which generally has lower values over urban areas, has been measured for TX data. It has been found that the full doppler spectrum has to be utilized for TX to generate a meaningful stationarity pixel map from SA coherency matrices. This analysis has been performed on TX fully polarimetric data acquired in April, 2009 over a site called Wallerfing in Germany.
\end{abstract}

\section{INTRODUCTION}

In SAR image processing, it is generally assumed that the sensor maintains a fixed look angle at the earth's surface, and also that the pulses are monochromatic. On the contrary, a SAR image is formed as a result of the integration of pulses from different look angles and wavelengths. Modern SAR sensors have a wide azimuth beam width and a large range bandwidth, but the effect of changes in azimuth angles and wavelengths on scene reflectivity are commonly ignored. These inaccurate assumptions may lead to incorrect analysis over urban areas, which consist of complex targets, having anisotropic structures, and exhibit a varying reflectivity over different azimuthal acquisitions [1]-[4]. Independent SAs can be used to detect non-stationarity, which has higher numerical values over urban areas compared to natural areas.

A 2D TF analysis to decompose polarimetric (pol) SAR images into their azimuth and range spectra for the detection of non-stationary pixels has been thoroughly explained in [3]. Although this analysis has already been performed on airborne SAR data, DLR's E-SAR at L-band [1], there are still no published results for its applicability to the space-borne case as mentioned in [2], [3]. The limiting factor for space-borne sensors is their comparatively small azimuth antenna aperture. In this paper, TF decomposition and urban area analysis using pixel non-stationarity has been evaluated for an X-band, spaceborne, fully pol SAR, TerraSAR-X. Throughout this paper TX Fully Polarimetric data will be referred to as TXFP data.

The rest of the paper is organized as follows. Section II gives a background of TF Decomposition, and pixel non- stationarity used for urban area analysis. Section III analyzes TF decomposition and non-stationarity of TXFP data, using different number of SAs and doppler bandwidths. Finally, sections IV and $\mathrm{V}$ briefly describe the results and list the conclusions, respectively.

\section{Time-Frequency Decomposition \& NON-STATIONARITY}

If $D(\omega)$ is the 2D Fourier tranform of a SAR image where $\omega=\left[\omega_{a z}, \omega_{r g}\right]$, and the subscripts az and rg represent azimuth and range, respectively, then the frequency response of the scene around a center frequency $\omega_{0}=\left[\omega_{0 a z}, \omega_{0 r g}\right]$ is given by [3]:

$$
D\left(\omega ; \omega_{0}\right)=D(\omega) G\left(\omega ; \omega_{0}\right)
$$

where $G\left(\omega ; \omega_{0}\right)$ is the 2D filter around the center frequency $\omega_{0}$. In Fourier domain SAR image signal can be represented as follows [3]:

$$
D(\omega)=R(\omega) H_{e}(\omega) H_{r}(\omega) W(\omega)
$$

where $R(\omega), H_{e}(\omega), H_{r}(\omega)$, and $W(\omega)$ correspond to the Fourier transforms of the scene coherent reflectivity, the emitted SAR signal, the focusing reference function, and the weighting function, respectively.

Before applying a 2D filter, spetral unweighting has to be applied on $D(\omega)$ to remove spectral imbalances introduced during SAR raw signal processing. This is achieved by calculating the average image amplitudes in range and azimuth, defined as the 2D weighting function, $W(\omega)$. The inverse of $W(\omega)$ is estimated and then applied to the 2D Fourier transform of the SAR image. Finally, the 2D filter, $G\left(\omega ; \omega_{0}\right)$, is applied to unweighted SAR image, and the inverse Fourier transform, $d\left(l ; \omega_{0}\right)$, of the result is calculated to get a SA image around a center frequency $\omega_{0}[3]$.

$$
d\left(l ; \omega_{0}\right)=F T_{2 D}^{-1}\left\{R(\omega) H(\omega) G\left(\omega-\omega_{0}\right)\right\}
$$

In this analysis, TF decomposition has been performed only in the azimuth direction with non-overlapping SAs. A TF analysis of airborne SAR images with application to urban areas has been presented in [5]. An important parameter computed between SA images is pixel stationarity, which is 
used to locate targets with anisotropic behavior across SAs. Moreover, it has been found that urban areas show a highly non-stationary behavior compared to natural areas [1].

It has been shown in literature that a sample n-look $3 \times 3$ coherency matrix, $\mathrm{T}_{3}$, follows a complex Wishart probability function, $\Sigma$ [3]. If the R SA sample $\mathrm{T}_{3 i}$ coherency matrices, $(i=1 \ldots R)$, of a pixel follow the same distribution, it is said to have a stationary spectral behavior. The following hypothesis needs to be satisfied in this case:

$$
\text { Hyp: } \Sigma_{1}=\Sigma_{2}=\Sigma_{3}=\ldots=\Sigma_{R}
$$

This hypothesis is tested by using a Maximum likelihood (ML) ratio $\Lambda$

$$
\begin{gathered}
\Lambda=\frac{\prod_{i=1}^{R}\left|\mathrm{~T}_{3 i}\right|^{n_{i}}}{\left|\mathrm{~T}_{3 t}\right|^{n_{t}}} \\
\text { where } \mathrm{T}_{3 t}=\frac{\sum_{i=1}^{R} n_{i} \mathrm{~T}_{i i}}{\sum_{i=1}^{R} n_{i}} \text { and } n_{t}=\sum_{i=1}^{R} n_{i}
\end{gathered}
$$

$n_{i}$ is the number of looks used to compute the sample $3 \times$ 3 coherency matrix $\mathrm{T}_{3 i}$. The hypothesis is accepted and the target is considered to be stationary if:

$$
\Lambda>c_{\beta} \quad \text { with: } \quad P_{f a}=P\left(\Lambda<c_{\beta}\right)=\beta
$$

where $P_{f a}$ is an arbitrarily chosen probability of false alarm, given in [2].

\section{TF ANALYSIS OF TERRASAR-X FULLY POLARIMETRIC DATA}

TXFP data (HH, VV, HV, VH), analysed in this paper has a resolution of $2.2 \mathrm{~m} \times 6.6 \mathrm{~m}(\mathrm{rg} \times \mathrm{az})$, and an azimuth bandwidth of $1380 \mathrm{~Hz}$ (C) DLR (2010). An optical snapshot of this data has been shown in fig. 1 and a pauli color coded RGB image showing the polarimetric channels $\left|S_{H H}-S_{V V}\right|$, $\left|S_{H V}\right|,\left|S_{H H}+S_{V V}\right|$ has been shown in fig. 2.

The feasibility of detecting non-stationary pixels for spaceborne, PolSAR sensors has been assessed by applying TF decomposition to TXFP data. The data has been decomposed into 3 and 5 SAs using half and full doppler spectrum, respectively. The reason to analyse half doppler spectrum is that it contains most of the spectral information of the scene. Figure 3 shows the magnitude of the average doppler spectrum of $\mathrm{S}_{H H}$ channel. For each TF decomposition, pixel stationarity $\Lambda$ has each been calculated. The first TF decomposition in the doppler bandwidth corresponds to approximately half of the doppler spectrum (1000 to $3000 \mathrm{~Hz}$ ), while the second decomposition comprises the full doppler spectrum (0 to 4000 $\mathrm{Hz}$ ). For an airborne SAR, characterized by a wide aperture and a high resolution in the azimuth direction (e.g. E-SAR has approx. $7.5^{\circ}$ of azimuth variation [3]), the half doppler spectrum might be suitable for TF decomposition. This limitation,

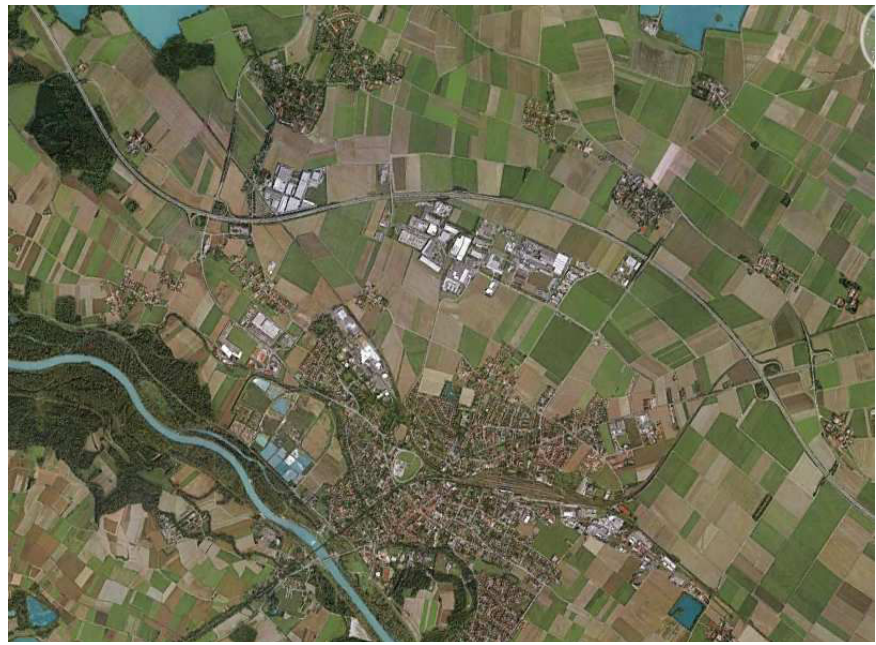

Fig. 1. Optical image (c) 2010 Google maps.

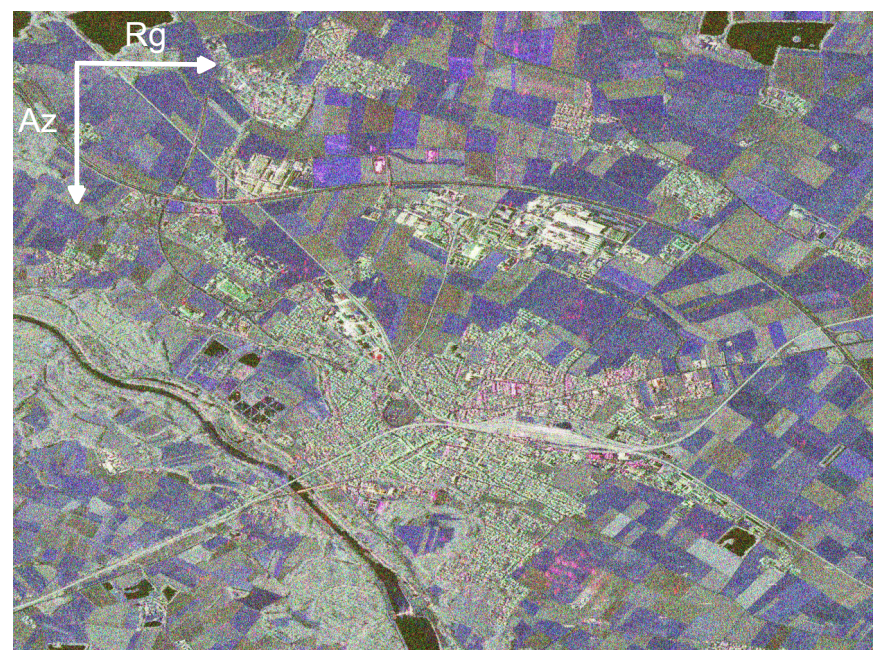

Fig. 2. Pauli color-coded image $\left|S_{H H}-S_{V V}\right|,\left|S_{H V}\right|,\left|S_{H H}+S_{V V}\right|$.

however, does not necessarily hold true in space-borne case. This is because space-borne SARs have a very small azimuth aperture and a significantly lower resolution. There exists a trade-off between the resolution in space and frequency domains, defined by the Heisenberg-Gabor uncertainty relation [3]:

$$
\Delta \omega \Delta l=\mu
$$

where $\Delta \omega$, and $\Delta l$ are the resolutions in frequency and spatial domains, respectively. A very fine resolution in frequency might produce a meaningless result in spatial domain due to a high degradation of spatial resolution.

TXFP data has an azimuth resolution of $6.6 \mathrm{~m}(\Delta l)$ and an azimuth bandwidth of $1380 \mathrm{~Hz}(\Delta \omega)$. If the full resolution image is decomposed into three independent SAs using half the doppler spectrum, then approximately half of the azimuth band width $(690 \mathrm{~Hz})$ is divided between the SAs, producing a fine frequency resolution of $230 \mathrm{~Hz}$, but in the spatial domain 


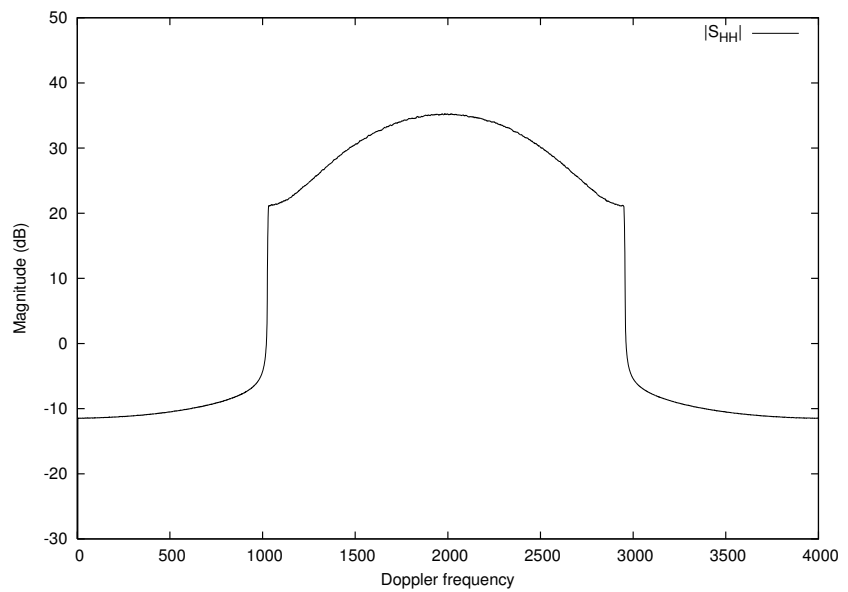

Fig. 3. Average doppler spectrum of $\left|S_{H H}\right|$.

the azimuth resolution degrades to approx. $39 \mathrm{~m}$. In contrast to this, for a high resolution airborne SAR, like E-SAR, the same procedure produces a spatial degradation from $1 \mathrm{~m}$ to only about $5 \mathrm{~m}$. The problem is solved by using the full doppler spectrum, which results in a much less degradation (approx. 19 $\mathrm{m})$. This analysis mandates the use of the full doppler spectrum for SA decomposition of TXFP data.

Pixel non-stationarity can be calculated using TF decomposition of TXFP data. However, unless the SAs are independent (non-overlapping), non-stationary pixel detection is a challenge. In the following section, pixel non-stationarity has been calculated and the results have been shown for half and full doppler spectrum using 3, and 5 non-overlapping SAs, respectively.

\section{RESUlts}

Figure 4 and 5 show $\Lambda$ for 3 and 5 SAs computed using half of the doppler spectrum. Brighter pixels show lower values of $\Lambda$. These figures do not reveal any useful information, in fact they look very random. On the other hand, figs. 6 and 7 show the same parameters using the full doppler spectrum. These figures show a significantly better separation of nonstationary pixels, and a visible improvement in the detection of urban areas as non-stationary pixels. Figures 8 and 10 show zoomed-in images of the areas selected in fig. 6 and 7, while fig. 9 shows their optical counterpart. A scrutinized comparison of figs. 8, 10 and fig. 9 shows a good identification of urban areas as non-stationary pixels. Of course, there are still some non-urban targets, which also exhibit non-stationary behavior. It has also been found that the decomposition into 3 non-overlapping SAs gives better results than 5 . This can be attributed to the fact that 5 independent SAs result in a poorer spatial resolution than 3 and it can be seen by comparing fig. 8 to fig. 10 . Notice, in contrast to fig. 8 , the non-stationary pixels in fig. 10 are blurred out and also merge into neighboring pixels.

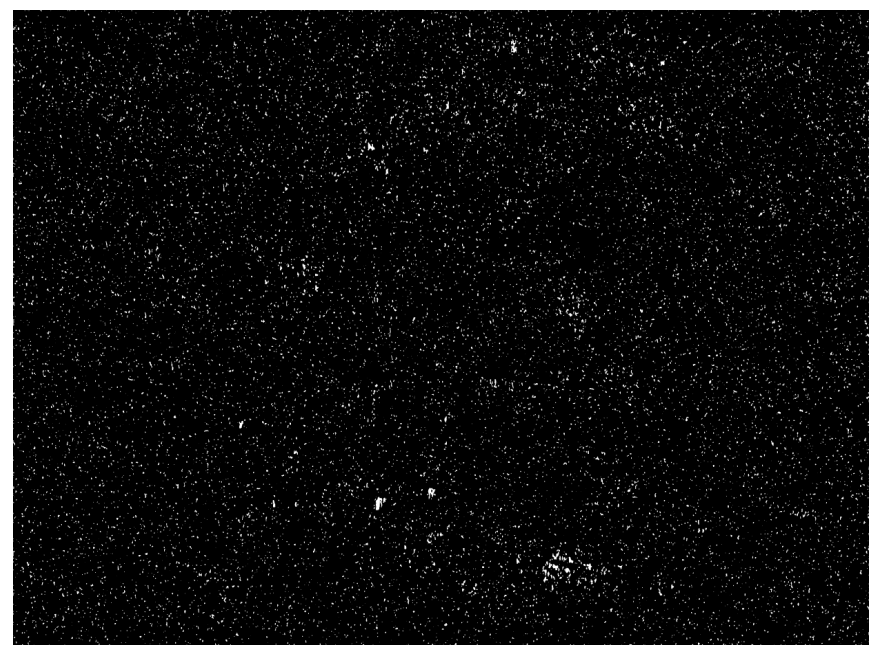

Fig. 4. $\Lambda$ using half doppler spectrum at $\mathrm{N}=3$.

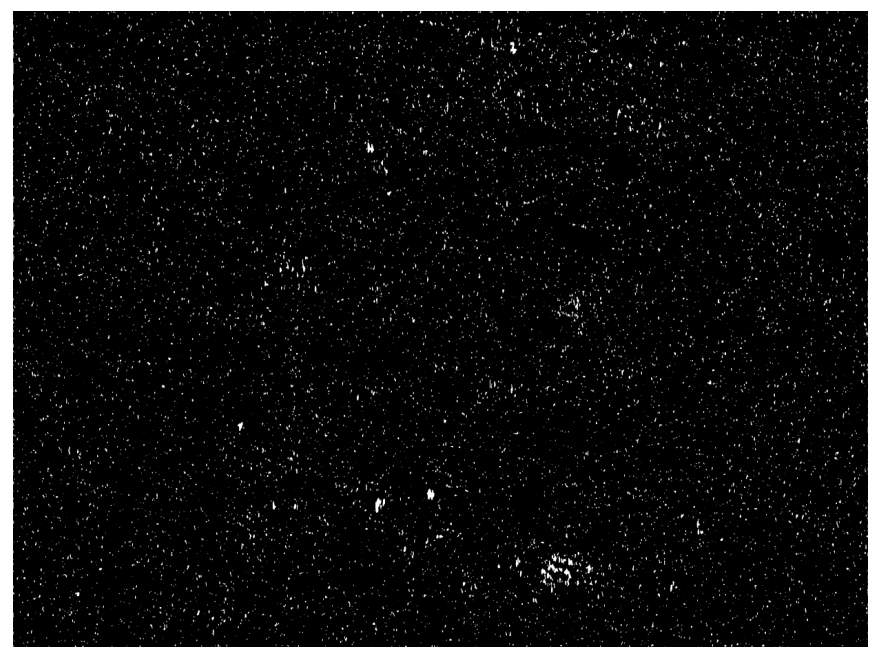

Fig. 5. $\Lambda$ using half doppler spectrum at $\mathrm{N}=5$.

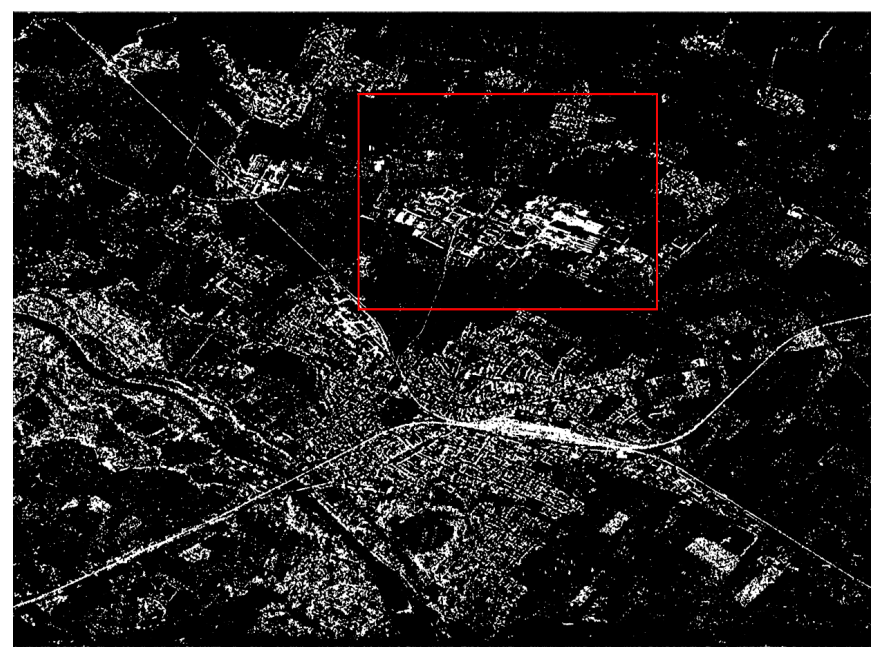

Fig. 6. $\Lambda$ using full doppler spectrum at $\mathrm{N}=3$ and the selected area (red rectangle). 


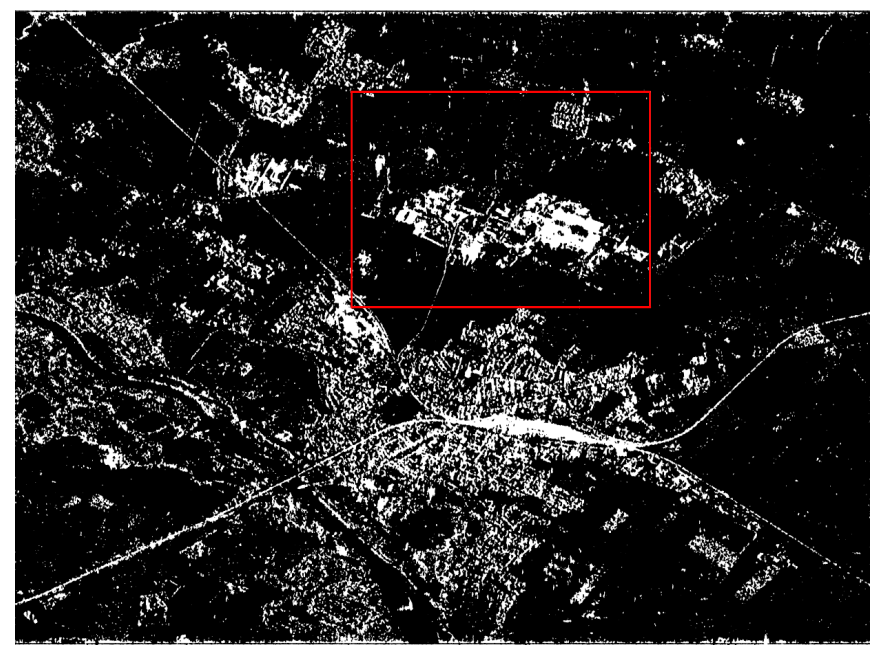

Fig. 7. $\Lambda$ using full doppler spectrum at $\mathrm{N}=5$.

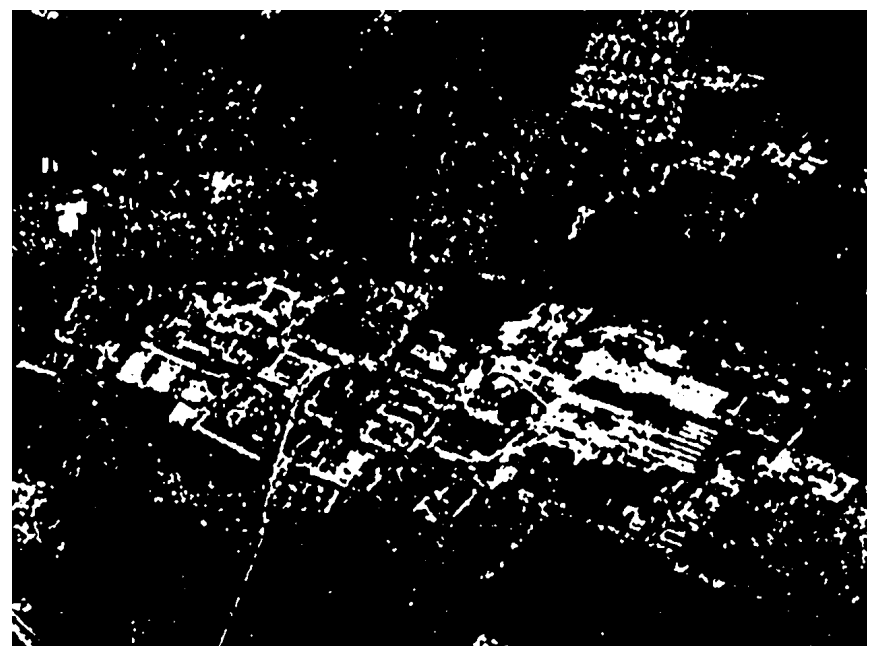

Fig. 8. Zoomed in $\Lambda$ using full doppler spectrum at $\mathrm{N}=3$.

\section{CONCLUSIONS}

In this paper, the feasibility of using TF techniques for the analysis of urban areas using fully polarimetric space-borne SAR sensor, Terrasar-X, has been investigated. The results show that for space-borne SARs, characterized by a small azimuth aperture, full doppler spectrum should be utilized for a meaningful SA decomposition. Pixel non-stationarity has been computed for TXFP data for different number of independent SAs over half and full doppler spectrum, respectively. It has been found that non-stationarity can be measured for TXFP data only if the full doppler spectrum is utilized. Nonstationarity is a useful measure, which can not only be used to detect urban areas, but it can also be utilized to remove SAs, which exhibit non-stationary behavior, from full resolution image [2]. The remaining stationary SAs can then form a (nearly) full resolution image without non-stationarities. This can contribute to a better estimation of geophysical properties from TXFP data.

In the future, similar analysis can be performed analyzing

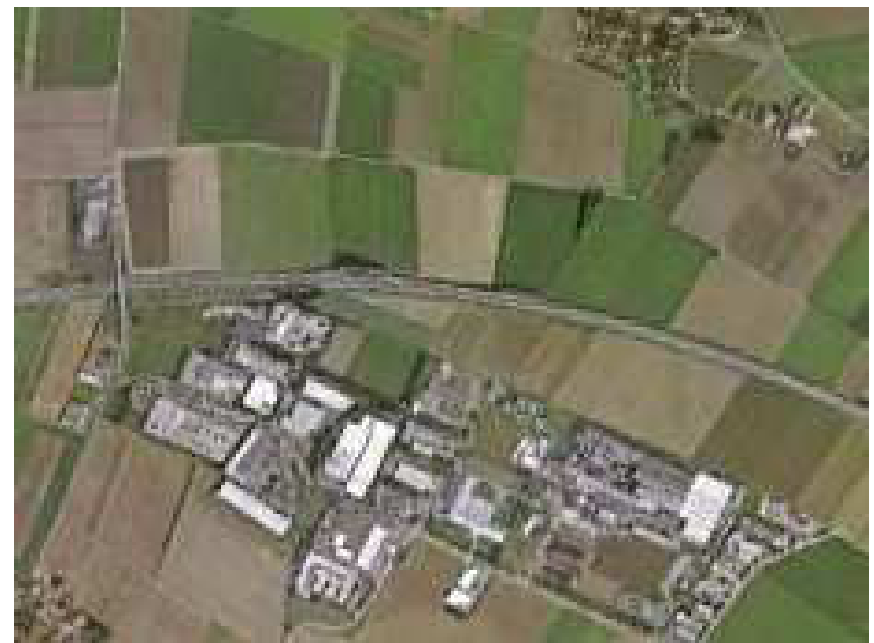

Fig. 9. Zoomed in optical image (c) 2010 Google maps.

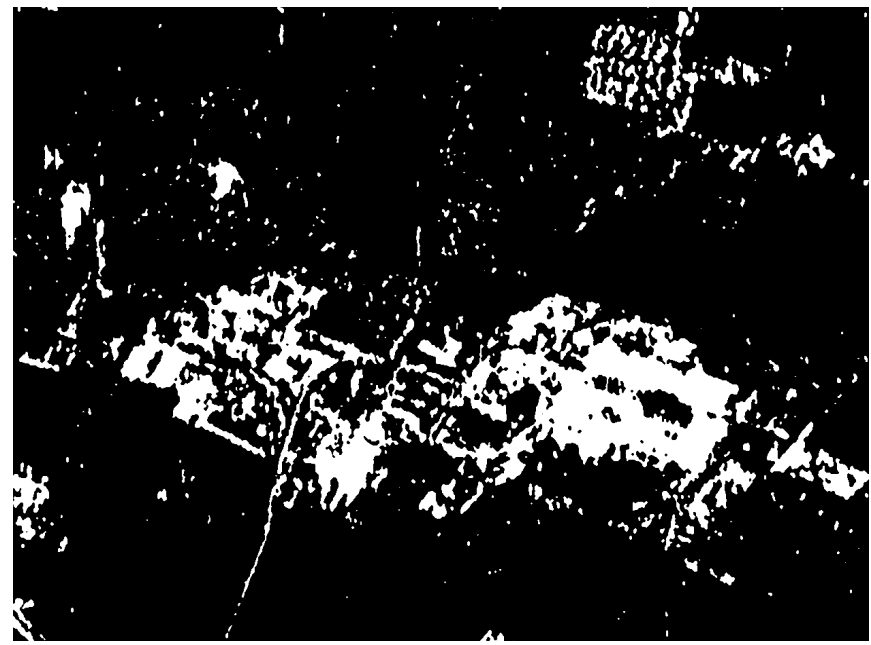

Fig. 10. Zoomed in $\Lambda$ using full doppler spectrum at $\mathrm{N}=5$.

the feasibility of decomposition in the range direction as well. Also, the recent announcement of opportunity to acquire TXFP data will allow the application of this analysis on more test data. Moreover, comparisons of this technique with other polarimetric decompositions can also be performed.

\section{REFERENCES}

[1] L. Ferro-Famil and E. Pottier. Urban area remote sensing from 1-band polsar data using time-frequency techniques. Urban Remote Sensing Joint Event, 2007, pages 1-6, april 2007.

[2] L. Ferro-Famil, A. Reigber, E. Pottier, and W.-M. Boerner. Scene characterization using subaperture polarimetric sar data. Geoscience and Remote Sensing, IEEE Transactions on, 41(10):2264 - 2276, oct. 2003.

[3] Eric Pottier Jong-Sen Lee. Polarimetric Radar Imaging: From Basics to Applications. CRC Press, 2009.

[4] A. Reigber, M. Jager, W. He, L. Ferro-Famil, and O. Hellwich. Detection and classification of urban structures based on high-resolution sar imagery. Urban Remote Sensing Joint Event, 2007, pages 1 -6, apr. 2007.

[5] P. Leducq, L. Ferro-Famil, and E. Pottier. Time-frequency analysis of polarimetric sar images. Radar Conference, 2005. EURAD 2005. European, pages $109-112$, oct. 2005. 